\title{
Philosophiques
}

\section{L'art et la norme, Société 15/16, Montréal, 1996, 464 p.}

\section{Suzanne Foisy}

Volume 25, numéro 2, automne 1998

Les modèles d'évolution en économie et en sciences sociales

URI : https://id.erudit.org/iderudit/027497ar

DOI : https://doi.org/10.7202/027497ar

Aller au sommaire du numéro

Éditeur(s)

Société de philosophie du Québec

ISSN

0316-2923 (imprimé)

1492-1391 (numérique)

Découvrir la revue

Citer ce compte rendu

Foisy, S. (1998). Compte rendu de [L'art et la norme, Société 15/16, Montréal, 1996, 464 p.] Philosophiques, 25(2), 300-304. https://doi.org/10.7202/027497ar

Ce document est protégé par la loi sur le droit d'auteur. L'utilisation des services d'Érudit (y compris la reproduction) est assujettie à sa politique d'utilisation que vous pouvez consulter en ligne.

https://apropos.erudit.org/fr/usagers/politique-dutilisation/ 
L'art et la norme, Société 15/16, Montréal, 1996, 16\%/ p.

Publié autour du Groupe interuniversitaire d'élude de la postmorlemité Montréali, Sociétć est un périodicque qui réfléchit sur les transformations sociales fondamentales et qui discute des "enjeux normatils " l'une sortie de la modernite. Notre intention n'est pas d'examiner tous les articles de ce recueil idont certains comportent nombre de pages ou d'établir la sempiternelle nature d'une esthétique postmoderne (qui ne s'y trouve pas. Nous aimerions cerner quelques questions autour du "lieu " actuel de l'esthétique dans le carrefour entre estlićticque et postmodernité. Les heureuses synthèses de L. Jacob (liminaire) viennent suppléer à l'ensemble hétéroclite. Il s'agissait, au départ, de clistinguer et d'articuler des niveaux d'analyse : 1) situation historique et ontologique de l'art ; 2) restructuration du système de l'art ; 3) tendances et enjeux de la pratique artistique. Parmi ces textes, deux projets se concentrent davantage sur la " normativité " de l'art. Ils feront l'objet de la majorité de nos commentaires et nous reviendrons ensuite très brièvement sur les autres contributions. 
Soucieux des présupposés d'une réflexion sur la "fragilité inhérente des oeuvres d'art „ dans le contexte de la crise actuelle, M. Ratté entend réfléchir sur la forme esthétique de la musique improvisée. Il lui importe d'évaluer les entreprises qui traitent de culture populaire d'un point de vue divergeant des idéologies qui polarisent l'institution savante de cette forme d'art vers l'axe moderniste/postmoderniste. La formalisation proposée veut élargir la portée de ces arts dont l'horizon esthétiçue a été voilé. Une nouvelle articulation de l'expérience de l'ceuvre par une critiçue immanente pourrait, en effet, contrer un postmodernisme qui négligerait la dialectique conslitutive du sens en abandomnant l'ceurre aux préconstructions de l'expérience médiatique. Le programme sociocomnunicationnel présenté veul saisir les modalités de reprobluction du sens de ees expériences el un espace d'interaction a priori qui devance l'arbilage de l'histoire de l'art. Colle reconstruetion des conditions d'une légitimité socioinstitutionnelle de la production artistique pratique une "anamnèse théorique " de la norme de surinterprétation de l'historicité afün de renouveler la compréhension de l'institution art comme "lieu d'émancipation de tous (normativité) el chacun (singularité)". Le concept paradigmatique de rationalité esthétique (Habermas, Wellmer, Secl, Rochlitz) qui a déjà fait valoir la spécificité de la modernité esthéticque en regard d'une raison élargie et spécifiée par l'expérience esthétique, est bonifié ici par un emboîtement de la dialectique de l'ouvre "dans la frange du communicable . Tandis que l'entreprise philosophique de Rochlitz (malgré son intérêt propre rate le sens des ceuves, l'unité pragmatique-communicationnelle et langagière de l'expérience esthétique chez Wellner n'a plus besoin de philosophie. Le contenu cognitif et l'ichange communicationnel des institutions artistiques se réalisent dans l'entrelacement des prétentions de validité dans le quotidien. Devant le constat d'un nanque de problématisation réciproque de la sphère de la communication banale et de colle de l'art et d'une abdication philosophique tant chez Wellmer que chez Habermas, l'A. propose que seule une réflexion conceptuelle et dialectique iqui opère la critique approfondie des weurres peut sonder l'efficacité de la rationalité communicationnelle vis-à-vis de la fluidification institutionnelle artisticue. Lorsque tous ces auteurs reprennent la critique arloniemne, la valeur normative d'un enracinement de l'art dans le vécu communicationnel n'est pas pensée dans le rapport aux auvres foù se tient la fragilité essentielle du sens. Á condition que les conceptions suivantes se débarrassent de leur référence à l'absolu, l'énoncé d'une phénoménologie de la création et de la réception qui postule la " normativité " de l'objel singuliel dhez Lukáes sympathise avec l'idée arlonienne d'une théorie matérielle ol matérialiste des formes qui réclame de la dialectigue un mime du nourenont immanent des aures. Ces eonjugaisons conduisent à transhigurer les conches communicationmelles réiliées.

Ine philosophic de lant a pesterion, qui meconfigurerait la coneption des expériences esthéliques en vue d'ébranler l'évidence de beur communieabilité, découvrimit les a sens esthéticues a d'Adorno, et chargimait sa eritique de la eulture que les eslbéticiens éludiés dans cel article n'auraient pas assumée. Celle "esthétique" aurait à repenser la zone grise de l'expérience communioationnelle et à amener la raison esthétique sur les voies (peu fréquenlées par l'esthélique actuellè d'un dialogue à la limite où, comme chez l'auteur de la Théorie esthétique, le sens s'exhale "à chacque fois ". Tout cela à l'occasion d'un " saut " qui n'est ni logique ni a priori et lors d'ume ouverture qui n'a rien à envier à l'intersubjeclivité consensuelle. "Autonomie de l'art et communication: le probleme de l'ouve d'art dans la thécrie sociocommunicatiomelle postadornienne", en plus de fournir un résumé-synthèse documenté et pesé de la problématique des esthétiques après Arlorno, témoigne d'une fidélité étonnante à Adorno par la suggestion même d'autres catégories esthétiques et par la critique des esthétiques qui le présupposent en le dépassant. Penser l'cuvre " comme une médiation symbolique complexe correspondant normativement à un intérêt pour la reproduction de l'expérience communicationnelle dans la frange du 
communicable" est cencs une avenue offerte aux recents développements "postadomiens "y compris ceux des " criteres esthétiques ". In retour final sur la frange du musical, alors que eette perspective avait inauguré efficacement la fomulation du début, aurait montré l'éclair de la pratique sur la théotie en supplément.

"La condition paradoxale de l'art dans la société postmorlene ", de M. Freitag, montre dans un copieux eent pages, non plus le privileze de la discipline philosophique, mais la prinaulé de la dimension esthélique. La première partie nous introduit à une phénoménologie de la " consubstantialité de l'être el de la forme ", où celle rlemière est le "moment premier du réel " qui devient la "matrice de l'ôtre "par sa a capacité immanente de transformation, d'enrichissement, de diversificalion, de nouvelle synthèse ". Mais cetle idée n'est concevable qu'en envisageant la genese de l'art moderne comme dimension sociale particularisée. La deuxiène partie met lout en ceuve pour caractériser l'ar moderne comme activité autonome et le parcours sociohistorifue de son folution depuis le Moyen Jure jusqu'au romantisme, oì il devient le lieu résenvé à la vérité de l'expressivité subjective, qui s'oppose à l'industrialisation, pour devenir une idéologie compensatoire. Cette a surenchère" dévoile pourtant lamore d'une "eondition paradoxale ". La modemilé esthétique " dernier soubresaut "de la modernité historicue porte en germe l'éclatement des paradigmes esthétiques. Nors qu'elle montrait l'activité globale de l'art iattiste of institution compris résistant aux activités de la réification, la postmodernité révèle la dissémination de la dimension expressive-normative hors de la sphère artistique. Devant cette perte de synthèse subjective, la troisième partie de l'article propose un projet philosophique (énigmatique) qui consiste à "cultiver le sens des formes "par l'intermédiaire desquelles une société peut s'onvir à l'exigence de ṣnthèse tréflexivité esthétique, normative et cognitive), tandis qu'elle devient, autrement, le "simple ensemble de ses systèmes ".

Loin des questionnements sur le statut des discours et des vagues esthétiques postmorlernes avec greffons philosophiques, ce texte -- à la rlifférene du précédent, plus centré sur l'expérience el la fonction esthétiques .... s"intéresse à lemplacement de l'esthétique dans la totalité sociale et couvre deux volets : le paradoxe de l'art et son rapport à la norme. Dans la modemité esthétique, la prégnance ontologique ne s'eleve à la conseience d'elle-même que par une dissociation épistémique, ontologique et sociale qui est d'abord a verticale et hiérarchicue ", pour se faire ensuite "horizontale et eatégorique n. Dans celte aporie qu’jl faut embrasser dans son déploiement historique el phénoménologique, on doit distinguer un versant philosephique et un versant sociologique. Le premier touche la réalisation de l'unité du sujet et de l'objet dans la forme sensible. Le paradoxe sociologique concerne le fait que l'art doit rechercher le rétablissement de cette synthèse, mais qu'il ne peut achever cette tâche: que dans une pratique sociale séparée. Il existe une lension entre l'iféalité esthélique et la réalité empirique que l'art nodeme parvient à résourle en se sabordant luimême, alors que le champ artistique continue de tenir en tension banalité et "vraie vie $"$.

La modernité présentait encore une coüncidence entre la spécilieité intérieure de l'art et sa constitution extérieure. Dans le passage à la postmodernité surgit un paradoxe d'un autre ordre (" hypothèse majeure " dans l'analyse de Freitag!: la manière clont il est perȩu socialement (concept classique) extérieur) ne correspond plus à l'idée de sa production (intérieur). Son authenticité contrarie de plus en plus son institutionnalisation en art world où le système se referme sur ses propres discours (Luhmann). C'est désormais au milieu de l'art lui-même que s'effectue la division entre sa surspécialisation et le monde de la vie et de l'expérience esthétique. Pour Freitag, cette mort de l'art coincide toutefois avec la redécouverte de l'importance de la 
synthèse sensible, d'où les dimensions cognitives et noimatives avaient été originellement (bien qu'arbitrairement) abstraites. Il incomberait à l'art d'entretenir l'a attente de beauté ". La rencontre entre art et norme s'établit dans la reconnaissance de la valeur cognitive et normative (objective inmanente) de la dimension esthétique qui fait le " pont "entre le cognitif et l'éthique et constitue leur " matrice " historique. La normativité est l'obligation que l'être contingent se donne pour perdurer dans sa forme et se déployer dans l'interaction. Or, l'art est conçu comme une " projection dans l'être objectif de l'universalité subjective "; il en est une "épiphanie ". Cette proposition de l'A. est affirmée sous l'angle d'un concept de postmodernité original : l'art morlerne n'est pas lant l'art spécilique de la modemité que l'art de la transition à la postmodernité. L'art postmodertue clevient par le fait mème un moment,

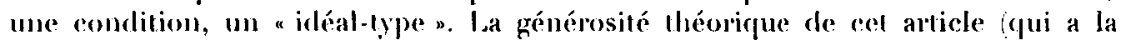
substance d'un live en complant les noles et les extraits prélevés semble devoir beaucoup à ces havres de réflexion que sont $\Lambda$. Portmann (1967), A. Pichot (1991), R. Williams 1966 et 1961) el M. Merleau-Ponty 1963). L' $\Lambda$., qui pratique la pensée dialectique doublée d'une culture phémoménologique, se donne en outre comme l'héritier de Kant (plus la dialectique ontologique hégélieme) et de Hegel (moins la métaphysique de l'absoluj. Malgré les nuances nombreuses qui rendent la lecture parfois difficile, et le mystère du projet d'une " cultivation " formelle (sa réinauguration relève de quelle instance?), ces lignes qui récapitulent et projettent sans cesse proposent une vision d'ensemble. La dimension esthétique appréhendée par le regard transdisciplinaire chez Freitag contraste avec la compétence exclusive attribuée à la philosophie chez Ratté. Mais l'enjeu est différent : l'un cherche le sens à distance de l'expérience, l'autre au-dedans.

On pourrait multiplier les affinités entre ces articles et les autres textes du numéro par duos et trios sur le thène du paradoxe de l'art et de sa normativité ¡Freitag/Laliberté/Côté/Dewitte; De Facendis/Ratté/Bellavance; ete). L. Jacob roit dans l'intescention de J. A. Maseolto une "poésie de circonstance ". "La postmodernité : un essai de clarification n, de J. Hoarau, reprend la pluralité des significations de ce concept en reconfirmant l'usage critique qu'en font Habermas et Freitag. "L'autonomic de l'art à l'ire de l'autonomie de tout ", de G. Bellavance, s'interroge sur la souveraineté de l'art dans le contexte de son institutionnalisation. "Faire son deuil de la mort des formes ", de R. Laliberté, approfondit le concept de déformalisation d'Elias en analysant une couvre littéraire de Segalen. Le domaine: esthélique s'y révèle comme le lieu d'inbrication des processus de déformalisation et d'individualisation. "Le sens ontologicue de l'omement ", de J. Dewitte, laate de l'art contemporain conme "symptôme et symbole de son ćpoque norecée s. En rélérence a Weidle et Sedlmayr oi du point de vue de la théche des valeurs de II. Broch, il clarilie l'essentiel du processus de purification interne ex externe de l'art aetuel ef son absence de signilication, avee le repère de l'architecture. "Panicpue daus la société. Eelgar Allan Poe el les origines de la modemilé esthétique anx Élats-tinis " lente de comprende l'émergenec de la modernité esthétique, en distinguant, avec Freilag, morlenité esthétique el modemité historique. Cluez J.-F. Côté, l'esthétique apparaît eomme ce site réflexif qui permet de poser el de régler des problèmes sociaux. La modernité esthétique fournirait à l'art un rôle différenciant dans un monde qui se clôt sur l'jdentité. Les textes de D. Vander Gucht (a Le devenir-monde du musée et le devenir-musée du monde ») a D. De Facendis (a Principes élémentaires d'une esthétique antibourgeoise ") - le premier plus sociologique, en présentant le problème d'une muséification de la mémoire sociale, le second plus philosophique, en problématisant la question esthétique par le biais du capitalisme - offrent des interprétations divergentes de la modernité esthétique relativement au problème de l'altérité. Le dernier est le pendant pessimiste des deux projets que nous avons d'abord rencontrés. L'atmosphère de critique sociologique qui flotte au-dessus de ce collectif 
permet d'envisager une esthétique plurielle, parfois inégale, qui clonne à penser l'art et son sens dans un cadre global que des disciplines plus pointues (par le haut on par te bas tendent à bannir carrément de leur's recherches. La constellation que cadence. l'article de Freitag at qui problématise les aspects cognitif's, expressifs et normatifs de l'expérience esthétique, où l'esthétique vient s'insérer oul s'échapper formme dimension, experience ou disciplinej, dévoile une large perspective. $\grave{A}$ la covisé des branches du savoir el en vertu de " laisons ", sociologie critipue el esthétigue conmu-

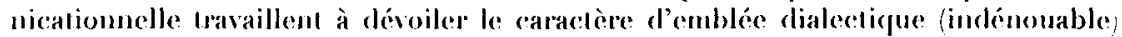
entre l'art et la société et, par conséquent, l'impossibilité de régler artificiellement la question esthéticue par une définition ou une surenchère.

Suzanne forsy

Département de philosophie

Université du Québec à Troris-Rivières 